\title{
Ultra-low density metallic foams synthesized by contact glow discharge electrolysis (CGDE) for laser experiments ${ }^{\star}$
}

\author{
Sandrine Rocher ${ }^{1, *}$, Ronan Botrel ${ }^{1}$, Frédéric Durut ${ }^{1}$, Cédric Chicanne ${ }^{1}$, Marc Theobald ${ }^{1}$, and Vincent Vignal ${ }^{2}$ \\ ${ }^{1}$ Commissariat à L'Énergie Atomique et aux Énergies Alternatives (CEA), VALDUC, 21120 Is-sur-Tille, France \\ 2 ICB, UMR 6303CNRS-Université de Bourgogne Franche Comté, BP 47870, 21078 Dijon Cedex, France
}

Received: 16 June 2017 / Received in final form: 22 September 2017 / Accepted: 5 December 2017

\begin{abstract}
The goal of this work is to realize metallic foams synthesized by contact glow discharge electrolysis with specific characteristics. In this paper, we show the results of our studies, consisting in investigating parameters that influence the foams characteristics. Thus, the morphology of metallic foams is examined through scanning electron microscopy (SEM) observations with the acid nature. Moreover, the evolution of the mass and the volume of metallic foams with two experimental parameters (overvoltage and gold concentration) is also investigated. The acid nature affects the foams microscopic structure highlighted by the SEM observations, but for now no valid explanation to this behaviour was found. We prove that the mass deposited on the electrode is dependent on the ionic salt concentration, whereas the overvoltage only affects the foam overall density.
\end{abstract}

\section{Introduction}

In order to study laser-matter interactions on the Megajoule Laser facility, CEA needs to synthesize metallic foams with specific characteristics. The goal is to realize in a millimetric scale, metallic foam samples with a relative density lower than $10 \%$ of the bulk material and structured with a micrometric porosity. However, the current processes developed to manufacture metallic foams such as gas injection [1-2], polymeric foam metallization [1-3] and so on cannot reach those characteristics all together. Thus, CEA has developed a new process using the contact glow discharge electrolysis technique (CGDE) to grow metallic foams at the cathode surface: both electrodes are immersed in the ionic solution in which the metallic salt is diluted, and the cathode is spinning round while the anode is still (Fig.1). Beyond laser target fabrication issues for laser matter experiments, this CGDE process is an original technological way to obtain innovative metallic foams which physical properties are interesting for various applications as for examples medicine, batteries or catalysis $[1,3,4]$. Under specific conditions and when a critical potential is applied called the breakdown voltage Uc, a uniform gaseous envelop is formed around the cathode immersed in the ionic solution. We here define another parameter called the overvoltage $\Delta \mathrm{U}$ corresponding to the difference between the applied voltage and the breakdown voltage. Due to the high electric field at the

\footnotetext{
‡ Contribution to the topical issue "Plasma Sources and Plasma Processes (PSPP)", edited by Luis Lemos Alves, Thierry Belmonte and Tiberiu Minea.

* e-mail: sandrine.rocher@cea.fr
}

electrode surface, sparks are formed between the cathode surface and the gas liquid interface. When they meet the liquid, they reduce metallic cations to form metallic strands with a bush-like shape, and ultra-low density foams are synthesized. This very fast process allows synthesizing $1 \mathrm{~mm}$ thick of foam in less than $10 \mathrm{~s}$. At the end of the synthesis, the cathode is removed from the solution while power is still supplying so that the foam on its surface remains dried in the gaseous envelope. Then, the foam is taken out of the electrode, weighted, its volume determined by measuring its outline shape, and its microscopic structure is observed by scanning electron microscopy (SEM). Its overall density is calculated with those two data. With uniaxial compacting of each foam and thermal treatment, a densification to the required relative density can be achieved. The aim of our work is to control the foam formation to produce a wide range of relative densities without compacting for several metallic foams $(\mathrm{Au}, \mathrm{Cu}, \mathrm{Pt}$, $\mathrm{Ag}$, alloys...). In this paper, the influence of the solution composition on the gold foam structure and overall density is presented. The different parameters we have investigated are the overvoltage, the acid nature and the ionic salt concentration.

\section{Experimental setup and characterization techniques}

Figure 1 represents schematically our process that synthesizes metallic foams on the cathode surface which is immersed in the ionic solution. The plasma is generated with a generator (Iso-Tech IPS 603) that supplies a direct current voltage at the cathode. The applied voltage depends on the electrolyte and ranges from 110 to $150 \mathrm{~V}$, 


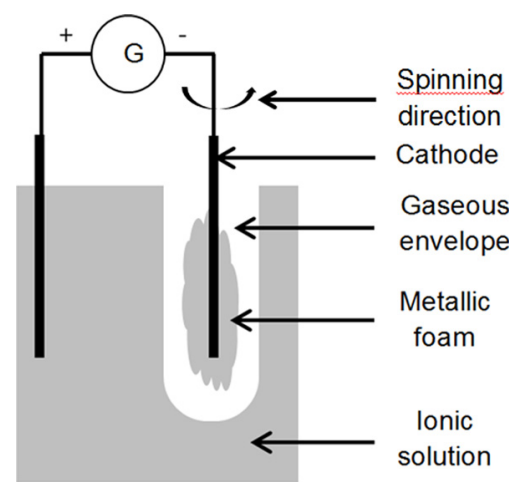

Fig. 1. Schematic process for the metallic foams synthesis.

with a power of $250 \mathrm{~W}$. The cathode is made of tungsten wire which diameter is around $1.0 \mathrm{~mm}$, the anode is a platinum grid, and the distance between the electrodes is around $3 \mathrm{~cm}$. In order to homogenize the foam growing on the electrode surface the cathode is spinning round at a speed of $1200 \mathrm{rpm}$ while the plasma is generated. The ionic solution is composed of tetrachloroauric acid $\mathrm{HAuCl}_{4}$ (Heraeus) diluted into distilled water and one acid which can be either sulphuric acid $\mathrm{H}_{2} \mathrm{SO}_{4}$ (Merck, $98 \%$ ), or nitric acid $\mathrm{HNO}_{3}$ (Sigma-Aldrich, 65\%) or hydrochloric acid HCL (Chem-Lab, 37\%).

The gold foams are characterized through their deposited mass (Mettler Toledo UMT2 accurate to $10^{-5} \mathrm{~g}$ ), their volume and thus their overall density is calculated with those two characteristics with a $2 \cdot 10^{-2} \mathrm{~g} /$ $\mathrm{cm}^{3}$ uncertainty. The volume is determined by an outline detector mostly made with a numerical camera, a rotating plate where the object is and an application created with Labview ${ }^{\circledR}$. This device detects the extern shape of the object over $180^{\circ}$ and calculates the inner volume, so we actually measure the foam envelope with a $4 \mathrm{~mm}^{3}$ accuracy. Besides, their microstructure is observed by SEM (Léo Carl Zeiss 1525). In this paper, each experiment was duplicated three times.

\section{Results and discussion}

\subsection{Effect of the acid nature}

The effect of three strong acids on the foams structures was firstly studied. Three solutions (1,2 and 3) were prepared. Solution 1 is composed of distilled water (A), tetrachloroauric acid at the concentration $60 \cdot 10^{-3} \mathrm{~mol} / \mathrm{L}(\mathrm{B})$ and nitric acid at the concentration $10 \mathrm{~mol} / \mathrm{L}$. Solution 2 is composed of A, B and hydrochloric acid $(10 \mathrm{~mol} / \mathrm{L})$, and solution 3 is made of $\mathrm{A}, \mathrm{B}$ and sulphuric acid $(10 \mathrm{~mol} / \mathrm{L})$. Gold foams were synthesized from those different solutions with the other experimental conditions kept constant, and their microstructure was observed by SEM. The pictures presented (Fig. 2a, b and c) have the same magnification.

First of all, for the same experimental conditions that is to say for the duplicated foams, the foams present the same microstructure showing the repeatability of our process. This structure in bush-like shape is notable as it is similar to the shape of a cathode discharge in liquid [5-7] that is why we believe that the foam grows directly on the plasma discharges during the process.

However, each acid leads to a different microscopic structure: the foams synthesized with nitric acid (Fig. 2a) have a structure really thin (the mean strands diameter is about $200 \mathrm{~nm}$ ), whereas the ones synthesized with hydrochloric acid (Fig. 2b) present nodular parts. Indeed, the strands are thin but present frequently bigger gold depositions. With sulphuric acid (Fig. 2c), the phenomenon is amplified, so the acid nature plays a significant role in the foam morphology.

The effect of the acid is quite hard to determine as no simple experiment can be set to give an explanation. Actually, referring to our hypothesis concerning the foam strand growth, we know that the shape and size of those discharges can be controlled by the solution conductivity [7-10]. The discharges in liquids with different conductivities are closely observed, especially their shape and structure. For a low conductivity, the discharge is thin and has a long propagation inside the liquid, whereas for a stronger conductivity the discharge is thicker and its propagation inside the liquid is shorter. In these experiments, the conductivity of the sulphuric solution is much higher $(650 \mathrm{~m} \mathrm{~S} / \mathrm{cm})$ than the conductivity of the nitric $(430 \mathrm{~m} \mathrm{~S} / \mathrm{cm})$ and hydrochloric $(390 \mathrm{mS} / \mathrm{cm})$ solutions that is why we observe such a shape evolution. Nevertheless, the conductivities of nitric and hydrochloric solutions are so close that it cannot explain the difference between the structures. At least one more phenomenon has to take place in this process to create these typical structures. We know that the chemistry at the interface is strongly changed with the formation of a lot of radicals and ions species strongly reactive [11-12] so it is possible that the reactions with the acids can be different and lead to those various structures. In order to confirm our hypothesis, we will soon observe the electrical discharges with a fast camera and compare their shapes with the foam strands.

\subsection{Effect of the gold concentration and overvoltage}

The effect of gold concentration on the foams characteristics was secondly studied. Three solutions were composed of sulfuric acid at the same concentration $10 \mathrm{~mol} / \mathrm{L}$, distilled water and tetrachloroauric acid at different concentration: $30 \cdot 10^{-3}, 60 \cdot 10^{-3}$ and $100 \cdot 10^{-3} \mathrm{~mol} / \mathrm{L}$. Those concentrations are respectively plotted on the graphi graphics (Fig. 3) as continuous, semi-dotted and dotted lines. Gold foams were synthesized from those different solutions with the same other experimental conditions, and several overvoltage were applied.

Figure 3a shows the results obtained about the foam masses synthesized with three solutions tested at different overvoltages. First, we notice that the overvoltage does not play a role on the foam mass as for similar conditions the foam mass remains constant whatever the overvoltage is. However, the deposited mass increases linearly with the gold concentration in the solution: the mass increase is about 190\% when the concentration doubles and about 


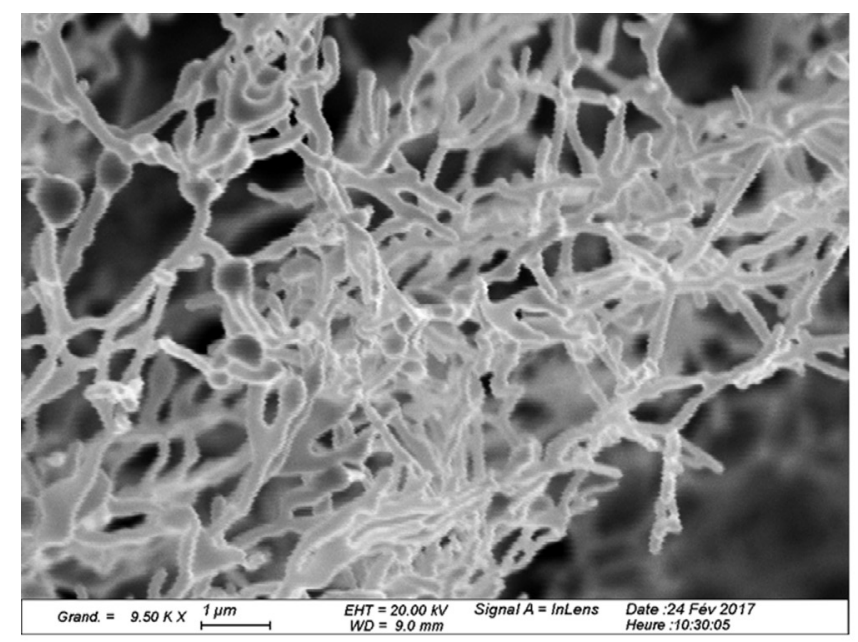

a)

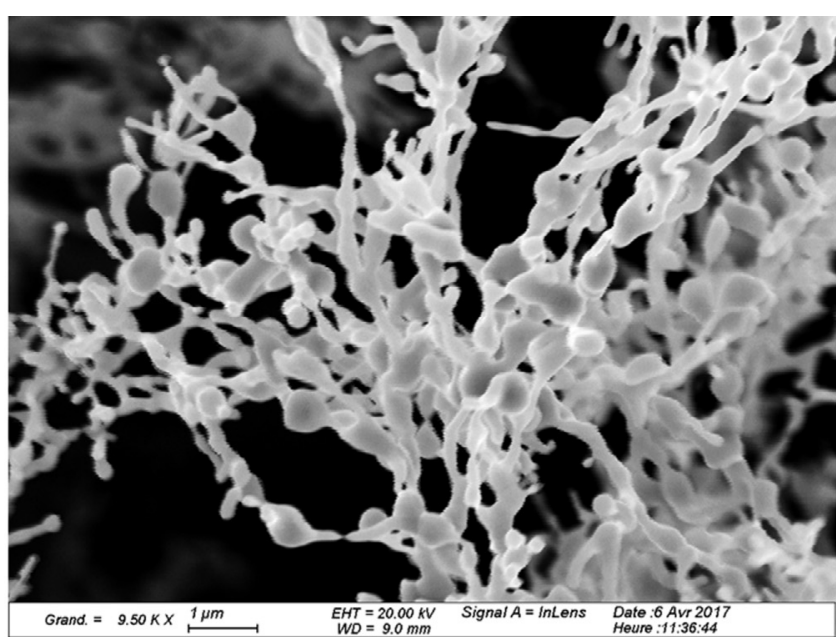

b)

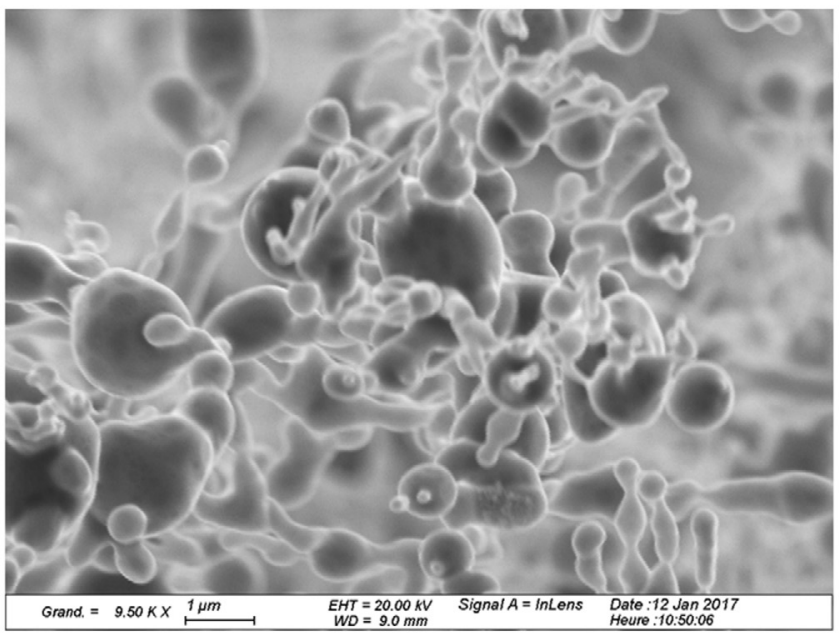

c)

Fig. 2. SEM pictures of gold foams synthesized from (a) solution 1 (nitric acid), (b) solution 2 (hydrochloric acid), (c) solution 3 (sulphuric acid).

$330 \%$ when the concentration triples. As the foam mass only depends on the ionic salt concentration and not on the energy supplied, that is to say only to the amount of metallic ions close to the interface, we can affirm that the foam deposition is driven by an electrolytic phenomenon.

Figure $3 \mathrm{~b}$ shows the volume measurement of those foams. While the dependency with the gold concentration is not really striking, it is clearly visible that the volume decreases when the overvoltage increases whatever the gold concentration is. Indeed, the decrease between the highest and the lowest overvoltage is about $35 \%$ for the two lowest concentrations, and around $60 \%$ for the highest one. We think that the size of the generated sparks increases leading to a denser foam.

Thus when the overall density is calculated from those data and the results plotted (Fig. 3c), it is logical that a dependency on both the ionic salt concentration and the overvoltage is observed. Hence, as the overvoltage increases, the overall density rises from 60 to $190 \%$ depending on the gold concentration. However, the overall density is not linear with the gold concentration because of the density mathematical definition which implies the volume variations. Finally, we obtain gold foams with relative densities between 0.2 and $1.1 \%$ of the bulk materials which are the weakest ever reached compared with the best results found in the literature for aluminum foams (2 to $30 \%$ ) [4,13], and for ferrous foams (35 to 45\%) [14]. To go further, we will soon investigate the effect of a different gold salt on the previous foams characteristics (deposited mass, volume and overall density).

\section{Conclusions}

The CGDE process is a satisfying and simple technique regarding the experimental conditions to produce ultralow relative density metallic foams very quickly. The mass deposition can be controlled by the gold concentration in the solution, and the foam volume by the overvoltage applied. A combined control of those two parameters leads to the foam overall density control. The microscopic structure is acid dependent but until now it can be roughly controlled as the complete mechanism is unknown. 


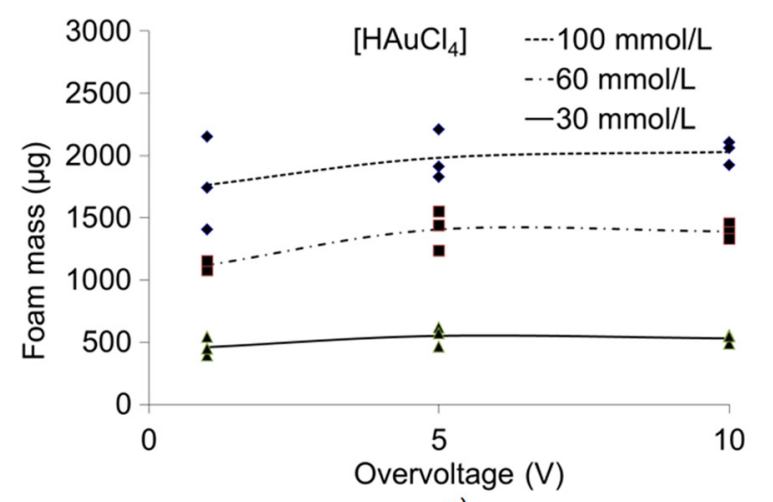

a)

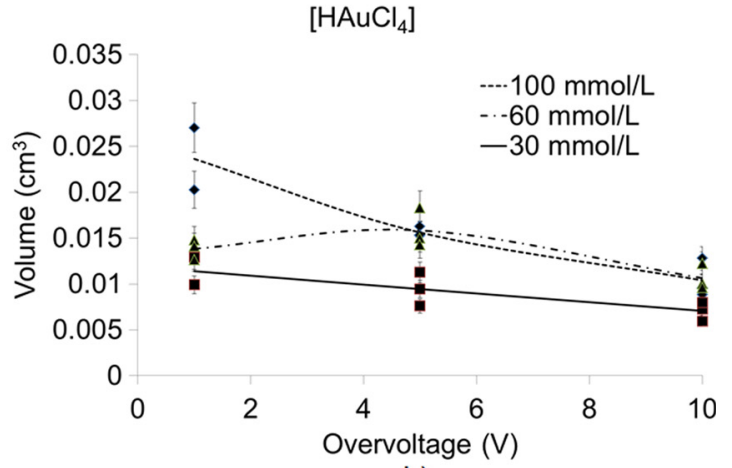

b)

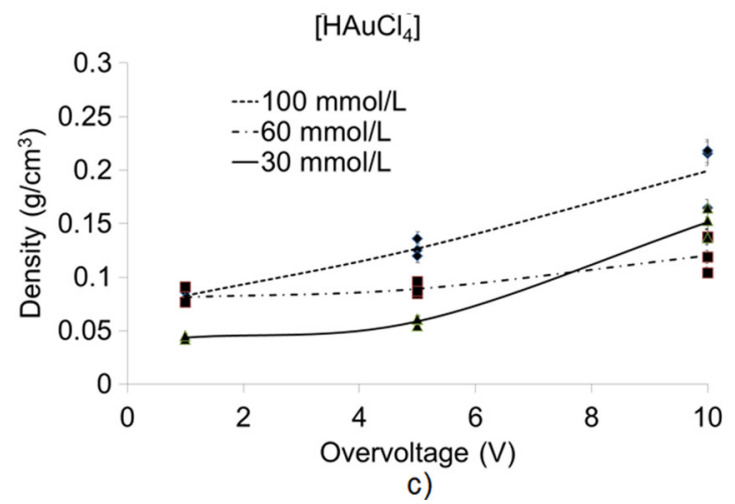

Fig. 3. Evolution of the characteristics (a) mass, (b) volume and (c) calculated density of several gold foams synthesized in similar conditions with the overvoltage and the gold concentration.

Further experiments have to be done for a better understanding of our process, especially the way of growing of the foams.

\section{References}

1. J. Banhart, Adv. Eng. Mater. 15, 3 (2013)

2. H. Haferkamp et al., Metal matrix composites and metallic foams, edited by T.W. Clyne, F.S. Simancik (Wiley, Weinheim, 2000)

3. A. Ochsner et al., Cellular and porous materials: thermal properties simulation and prediction, edited by A. Oschner, G.E. Murch, M.J.S. de Lemos (Wiley, Weinheim, 2008)

4. C. Körner et al., Metal matrix composites and metallic foams, edited by T.W. Clyne, F.S. Simancik (Wiley, Weinheim, 2000)

5. V.Y. Ushakov et al., Impulse breakdown of liquids (Power systems), edited by V.Y. Ushakov (Springer, Berlin, 2007)

6. J.F. Kolb et al., J. Phys. D: Appl. Phys. 41, 234007 (2008)
7. P. Ceccato, Ph.D. thesis, Paris-Saclay University, 2010

8. P. Bruggeman et al., J. Phys. D: Appl. Phys. 42, 053001 (2009)

9. Z. Kozakova, Ph.D. thesis, Brno University of Technology, 2011

10. S. Gasanova, Ph.D. thesis, Duisburg-Essen University, 2013

11. A.M. Lietz et al., J. Phys. D: Appl. Phys. 49, 425204 (2016)

12. J. Franclemont et al., J. Phys. D: Appl. Phys. 48, 424004 (2015)

13. C. San Marchi et al., Metal matrix composites and metallic foams, edited by T.W. Clyne, F.S. Simancik (Wiley, Weinheim, 2000)

14. U. Mohr et al., Metal matrix composites and metallic foams, edited by T.W. Clyne, F.S. Simancik (Wiley, Weinheim, 2000)

Open Access This article is distributed under the terms of the Creative Commons Attribution License https://creativecom mons.org/licenses/by/4.0 which permits unrestricted use, distribution, and reproduction in any medium, provided the original author(s) and source are credited.

Cite this article as: Sandrine Rocher, Ronan Botrel, Frédéric Durut, Cédric Chicanne, Marc Theobald, Vincent Vignal, Ultra-low density metallic foams synthesized by contact glow discharge electrolysis (CGDE) for laser experiments, Eur. Phys. J. Appl. Phys. $\mathbf{8 1}, 10803(2018)$ 